TP Periodica Polytechnica Electrical Engineering and Computer Science

\author{
58(4), pp. 149-159, 2014 \\ DOI: 10.3311/PPee.7806 \\ Creative Commons Attribution (i)
}

RESEARCH ARTICLE

\section{Time Optimal Control of Four-in-Wheel-Motors Driven Electric Cars}

\author{
György Max ${ }^{1 *} /$ Béla Lantos
}

ReCeived 10 November 2014; Accepted 2 January 2015

\begin{abstract}
The paper deals with the time optimal control of automatically driven electric cars in a test path under state and input constraints. The problem can be formulated as a dynamic nonlinear optimal control problem (DNOCP). The resulting $D N O C P$ is solved by reformulating it to a static nonlinear program (NLP) using time discretization and direct multiple shooting methods. A novel method is presented to convert the optimal solution obtained using the single-track model to the optimal control of four-in-wheels-motors driven (4WD) cars. The conversion assures similar motion of the COG of both models and optimal distribution of the longitudinal wheel forces. A discrete model predictive control (MPC) is proposed for the linearized $4 W D$ vehicle model under perturbations which uses the distributed wheel forces and optimizes the perturbations with analytically solvable end constraints. The elaborated method can form the basis to generate an offline database of a general collision avoidance system (CAS).
\end{abstract}

\section{Keywords}

4 WD electric vehicle $\cdot$ Time optimal control $\cdot$ Direct multiple shooting $\cdot$ Nonlinear programming $\cdot$ Optimal force distribution - Model predictive control

\footnotetext{
${ }^{1}$ Department of Control Engineering and Information Technology, Budapest University of Technology and Economics, H-1117 Budapest, Magyar Tudósok krt. 2., Hungary

* Corresponding author, e-mail: max@iit.bme.hu

${ }^{1}$ Research Centre of Vehicle Industry,

Széchenyi István University,

H-9026 Györ, Egyetem tér 1., Hungary

e-mail: lantos@iit.bme.hu
}

\section{Introduction}

Pursuit of optimal behavior in technical and other (biological, economical etc.) type of systems is a common goal of human research. In this area dynamic and static optimization problems are of different complexity. Especially, the difficulty increases if some of the optimization variables are integer or binary valued as in the case of combustion engine driven cars where the gear shift in the control is an integer variable. Solution of such MIOCP (mixed-integer optimal control) problem was presented in an earlier work [1]. Similar problems were discussed in the literature for cars described by ordinary (ODE) or differential-algebraic (DAE) equations in [2-4] considering fixed or moving time interval or based on moving horizon predictive approaches $[5,6]$.

There are several techniques to solve dynamic nonlinear optimal control (DNOCP) problems: dynamic programming approach by solving the Hamilton-Jacobi-Bellmann equation, indirect methods (known as first optimize, then discretize) and direct methods (first discretize, then optimize). The latter group of techniques can be applied to large-scale optimal control problems. In direct methods the continuous time infinite dimensional DNOCP problem is first discretized and reformulated to a finite-dimensional static nonlinear program (NLP).

A professional software package to solve the original continuous time problem may be MUSCOD-II [7], however it is not an open software. Hence, the novel professional and open optimization system OPTI [8] was chosen, which has also an interface to AMPL modeling language to formulate optimum problems at high level and has better performance than MATLAB Optimization Toolbox.

A novel method will be presented to solve the time optimal control of electric cars first using a single track dynamic model, i.e. the time optimal control of two-in-wheel-motors driven (2WD) cars, in order to decrease the complexity of the optimization problem. In the discussion the contact forces between tire and road will be modeled using Pacejka's magic formulas which assure more accurate modeling than using cornering stiffnesses and linearization. The reason is that time optimality results in large acceleration/deceleration forces leaving the linear domain of the slip angle and force characteristics. 
Having solved the 2WD time optimal control task, the next step is the optimal distribution of the control forces for the four-in-wheel-motors driven (4WD) case. The distribution of longitudinal forces has a large influence on vehicle handling characteristics such as the driver/vehicle interaction, road holding and yaw stability, in particular during combined traction/ braking and cornering near the grip limit of the tires. In order to select and develop suitable active control that provide consistent driver/vehicle handling, maximum road holding and sufficient yaw stability margins, it is essential to understand the influence of a particular drive force distribution on these handling characteristics.

Due to the highly non-linear interaction between the longitudinal and lateral forces near the grip limit, the studies in this area have thus far focused on prototype testing and/or simulations with sophisticated vehicle models. From the recent results we refer here to some works.

Sawase and Ushiroda [9] described the calculation of vehicle dynamics improvement by means of right-and-left torque vectoring system in various types of drive trains and analyzed the effectiveness for front wheel drive (FWD) and rear wheel drive (RWD) vehicles based on the tire maximum friction circle.

Ono et al. [10] proposed a method for 4W distributed steering and $4 \mathrm{~W}$ distributed traction/braking system based on friction circle of each wheel. The distribution algorithm used SQP (Sequential Quadratic Programming) for calculating the magnitude and directions of tire forces which satisfy constraints corresponding the resultant force and moment of the vehicle motion and maximize each tire grip margin.

Klomp [11] developed a method for the distribution of the longitudinal tire forces using quadratically constrained linear programming (QCLP) in order to maximize the global force in a predetermined direction relative to the longitudinal direction of the vehicle.

We have investigated all these methods and stated that their effectiveness is not satisfactory for high speed vehicle motion between corridors. Hence, we present a novel method that is based on linearly constrained quadratic programming (LCQP) and assure similar motion of the 2WD and 4WD car.

Unfortunately, the initial state of the car typically differs from those of the 2WD time optimal solution, hence a quick real time method is needed to reduce the effect of differences in the initial states. For this purpose 4WD moving horizon model predictive control (MPC) will be suggested which tries to eliminate the error at the end of each horizon and can be solved analytically using Lagrange multiplier technique. The method can tolerate the differences in the initial state while saving the form of the optimal trajectory after a short transient. Using this method, limits of the control forces can not be considered but fortunately the transient errors are quickly decaying and there is good chance to take into consideration the constraints. If it would be critical then MPC can be formulated as a Quadratic
Programming (QP) under state and control constraints which can be converted to a problem solvable by OPTI.

The novel methods can be integrated to a Collision Avoidance System (CAS) which starts using the database of offline computed and stored time optimal control solutions over a parameter grid, then the system selects the nearest optimal one for the actual traffic situation, performs optimal force distribution, exploits the tolerance in the initial state and realizes nearoptimal behavior of the CAS system.

In the sequel it will be assumed that robust servosystems are available for the wheels for which the longitudinal force limit can be converted to torque limit (based on the effective tire radius) and this limit is available for the optimization.

The structure of the paper is as follows. In Section 2 the time optimal control problem of the single-track vehicle is formulated and it is solved in Section 3 by the multiple shooting method using time, state and control discretization with the novel algorithm of computing the derivatives of the complex trajectory joining constraints for the Jacobians. Section 4 describes the method of the 2WD optimal wheel force distribution to the four-wheel driven vehicle. In Section 5 the distributed control forces of the 4WD vehicle are used in the moving horizon predictive control scheme in order to tolerate differences in the initial states. Numerical results of the problems are given in the corresponding sections. Finally, the paper is concluded in Section 6.

\section{Concept of Time Optimal Control Problem}

\subsection{Single-track vehicle model}

Consider an electric car moving in a horizontal plane driven by the rear wheels and steered by the front wheels. It is assumed that the right $(r)$ and left $(l)$ side of the vehicle is symmetrical, thus the two halves can be merged to a single-track model, see Fig. 1. In this paper, only the planar motion of the vehicle is considered. This means that other dynamics such as rolling, pitching of the vehicle are neglected.

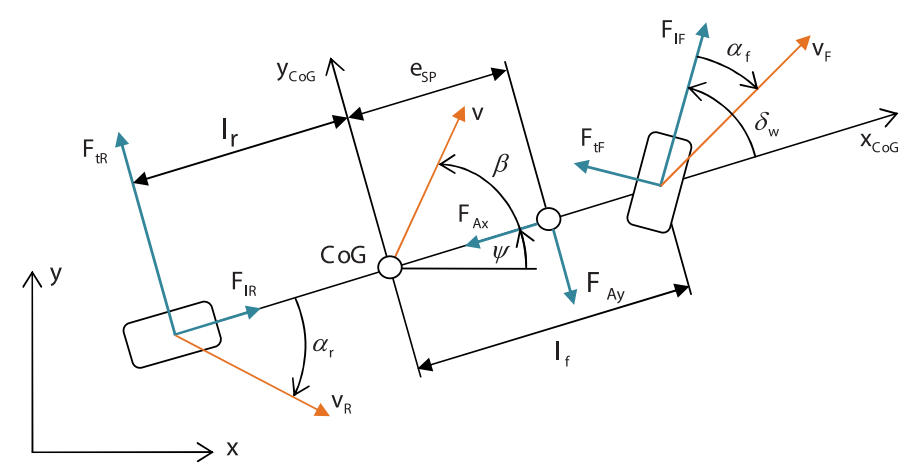

Fig. 1. Single-track model of vehicle

The states of the vehicle model are $p_{x}, p_{y}, v, \delta_{w}, \beta, \psi, \omega_{z}$, i.e. the $\mathrm{x}-\mathrm{y}$ position of the center of gravity $(\mathrm{CoG})$, the magnitude of velocity, steering angle of the front wheel, the side-slip angle, 
the orientation and the yaw rate respectively. The control variables that represent the driver's input to the vehicle are denoted with $\omega_{\delta}, U_{F}, U_{R}$, which are respectively, the angular velocity of the steering wheel and the front and right longitudinal control force (traction and braking) due to the engines' torques.

The single-track dynamics of the car can be given in the form of $\dot{x}=f_{2 W}(x, u)$ by the following system of ordinary differential equations (ODE) similar in [12] and [13]:

$$
\begin{gathered}
\dot{p}_{x}=v \cos (\psi+\beta) \\
\dot{p}_{y}=v \sin (\psi+\beta) \\
\dot{v}=\frac{1}{m}\left[\left(F_{l R}-F_{A x}\right) \cos (\beta)+F_{l F} \cos \left(\delta_{w}-\beta\right)\right. \\
\left.+\left(F_{t R}-F_{A y}\right) \sin (\beta)-F_{t F} \sin \left(\delta_{w}-\beta\right)\right] \\
\dot{\delta}_{w}=\omega_{\delta} \\
\dot{\beta}=\frac{1}{m v}\left[\left(F_{A x}-F_{l R}\right) \sin (\beta)+F_{I F} \sin \left(\delta_{w}-\beta\right)\right. \\
\left.+\left(F_{t R}-F_{A y}\right) \cos (\beta)+F_{t F} \cos \left(\delta_{w}-\beta\right)\right]-\omega_{z} \\
\dot{\psi}=\omega_{z} \\
\dot{\omega}_{z}=\frac{1}{I_{z z}}\left[F_{t F} l_{f} \cos \left(\delta_{w}\right)-F_{t R} l_{R}-F_{A y} e_{S P}\right. \\
\left.+F_{l F} l_{F} \sin \left(\delta_{w}\right)\right]
\end{gathered}
$$

with state and control variable vectors of

$$
x=\left(p_{x}, p_{y}, v, \delta_{w}, \beta, \psi, \omega_{z}\right)^{T}, u=\left(\omega_{\delta}, U_{F}, U_{R}\right)^{T}
$$

External forces $F_{t}, F_{l}, F_{A}$, are the transversal, longitudinal and aerodynamic forces acting on the car respectively. The second subscripts $R, F$ and $x, y$ indicate the rear, front wheel and the direction of the aerodynamic force respectively.

The rolling resistance of the front and rear wheel is computed from the friction as a velocity dependent function and the static load distribution, see [5], as follows:

$$
\begin{gathered}
f_{r}(v)=9 \cdot 10^{-3}+7.2 \cdot 10^{-5} v+5.038848 \cdot 10^{-10} v^{4} \\
F_{r F}=f_{r}(v) \frac{m l_{R} g}{l_{F}+l_{R}} \quad F_{r R}=f_{r}(v) \frac{m l_{F} g}{l_{F}+l_{R}}
\end{gathered}
$$

The total longitudinal wheel forces consist of the control forces $U_{F}, U_{R}$ and the rolling resistances

$$
F_{l j}=U_{j}-F_{r j}, \quad j=\{F, R\}
$$

The transversal (lateral) forces are described by Pacejka's magic formula, see in [14] and [15]. The front and rear slip angles and transversal forces can be given by

$$
\alpha_{f}=\delta_{w}-\arctan \left(\frac{l_{f} \dot{\psi}+v \sin \beta}{v \cos \beta}\right)
$$

$$
\begin{gathered}
\alpha_{r}=\arctan \left(\frac{l_{r} \dot{\psi}-v \sin \beta}{v \cos \beta}\right) \\
F_{t F, t R}=D_{f, r} \sin \left(C _ { f , r } \operatorname { a r c t a n } \left(B_{f, r} \alpha_{f, r}-\right.\right. \\
\left.\left.E_{f, r}\left(B_{f, r} \alpha_{f, r}-\arctan \left(B_{f, r} \alpha_{f, r}\right)\right)\right)\right)
\end{gathered}
$$

It is assumed that only longitudinal drag force acts on the vehicle (i.e. no side wind). Thus, the aerodynamic force due to air resistance can be determined by

$$
F_{A x}=\frac{1}{2} c_{w} \rho A v^{2}, \quad F_{A y}=0
$$

The numerical parameters of the dynamical model are from [3] and contained in Table 1.

\subsection{Test path}

A test path between corridors is considered as a double-lane change maneuver. The driver is required to overtake a static obstacle by changing and returning to the car's initial lane. The corridor is defined by twice differentiable cubic polynomials and the lower $P_{l}(x)$ and upper $P_{u}(x)$ path boundaries are shown in Fig. 4. For safety reasons, the car is restricted to move in the region of $B=0.84 \mathrm{~m}$ half track width vertically from the path boundaries. Details on similar test path can be found in [16].

\subsection{Dynamic optimal control problem}

The aim is to drive the car as fast as possible through the test path by maintaining a smooth comfort level (i.e. minimal steering effort). A natural objective function to this problem may be to minimize the total time $t_{f}$ needed to complete the path and regulate the driver's input $\omega_{\delta}$. Thus, the resulting dynamic nonlinear optimal control problem (DNOCP) can be read as

$$
\begin{gathered}
\min _{x(\cdot), u(\cdot), t_{f}} t_{f}+\int_{0}^{t_{f}} \omega_{\delta}^{2}(t) d t \\
\text { s.t. } \quad \dot{x}(t)=f_{2 W}(x(t), u(t)) \\
p_{y}(t) \in\left[P_{l}\left(p_{x}(t)\right)+B, P_{u}\left(p_{x}(t)\right)-B\right] \\
p_{x}(t) \in[0,170] \\
v(t) \in[10,40] \\
\delta_{w}(t) \in[-0.5,0.5] \\
\beta(t) \in[-0.2,0.2] \\
\psi(t) \in[-\pi / 2, \pi / 2] \\
\omega_{z}(t) \in[-1,1] \\
\omega_{\delta}(t) \in[-0.5,0.5]
\end{gathered}
$$




$$
\begin{aligned}
& U_{F}(t), U_{R}(t) \in\left[-10^{4}, 10^{4}\right] \\
& x\left(t_{0}\right)=(0, \text { free }, 10,0,0,0,0) \\
& p_{x}\left(t_{f}\right)=170, \quad \psi\left(t_{f}\right)=0
\end{aligned}
$$

where $f_{2 \mathrm{~W}}$ is the ODE system described in (1). The path boundary conditions are formulated in $(9 \mathrm{c})$, the constraints on the states and control inputs are given in $(9 d-9 i)$ and $(9 j-9 k)$ respectively. Initial and final values are defined by Eqs. (91) and (9m). Notice, that the initial vertical position of the car can be

\begin{tabular}{|c|c|c|c|}
\hline & Value & Unit & Description \\
\hline$m$ & $1.239 \times 10^{3}$ & $\mathrm{~kg}$ & mass of car \\
\hline$g$ & 9.81 & $\mathrm{~m} / \mathrm{s}^{2}$ & gravity constant \\
\hline$I_{\mathrm{F}}$ & 1.190 & $\mathrm{~m}$ & front wheel distance to cog \\
\hline$I_{\mathrm{R}}$ & 1.375 & $\mathrm{~m}$ & rear wheel distance to cog \\
\hline$b_{f}$ & 0.84 & $\mathrm{~m}$ & front half track width \\
\hline$b_{\mathrm{r}}$ & 0.84 & $\mathrm{~m}$ & rear half track width \\
\hline$I_{z z}$ & 1752 & $\mathrm{~kg} \times \mathrm{m}^{2}$ & moment of inertia \\
\hline$\rho$ & 1.249512 & $\mathrm{~kg} / \mathrm{m}^{3}$ & air density \\
\hline$A$ & 1.4378946874 & $\mathrm{~m}^{2}$ & effective flow surface \\
\hline$e_{\mathrm{SP}}$ & 0.5 & $\mathrm{~m}$ & drag mount distance to cog \\
\hline$c_{\mathrm{w}}$ & 0.3 & & air drag coefficient \\
\hline$B_{\mathrm{f}}$ & 10.96 & & Pacejka parameter (stiffness) \\
\hline$B_{r}$ & 12.67 & & Pacejka parameter (stiffness) \\
\hline$C_{f, r}$ & 1.3 & & Pacejka parameter (shape) \\
\hline$D_{\mathrm{f}}$ & 4560.4 & & Pacejka parameter (peak) \\
\hline$D_{\mathrm{r}}$ & 3947.81 & & Pacejka parameter (peak) \\
\hline$E_{f, r}$ & -0.5 & & Pacejka parameter (curvature) \\
\hline
\end{tabular}
chosen freely. All dimensions are in SI units.

Tab. 1. Parameters used in the vehicle models

\section{Implementation strategies}

In this section the control design possibilities based on existing software systems are discussed and the novel design strategy is presented. To solve the time-optimal problem introduced in Section 3, the application of OPTI Toolbox [8] and the noncommercial, open source solver IPOPT [17] was preferred. For further details on these software systems see [18] and [19].

The standard form of nonlinear program (NLP) that can be solved by using OPTI is of type

$$
\begin{gathered}
\min _{x} \quad f(x) \\
\text { s.t. } \quad A x \leq b \\
A_{\mathrm{eq}} x=b_{\mathrm{eq}} \\
c(x) \leq d \\
c_{\mathrm{eq}}(x)=d_{\mathrm{eq}}
\end{gathered}
$$

$$
\begin{gathered}
l_{b} \leq x \leq l_{u} \\
x_{i} \in \mathbb{R}
\end{gathered}
$$

In order to solve the dynamic optimization problem (9), it has to be transformed into the form of (10) using control and state discretization, see [6].

\subsection{Discretization for varying final time}

The final time varies, thus discretization for normalized interval $[0,1]$ has to be performed in order to make the controls and states independent from $t_{f}$. The time transformation is

$$
t(\tau)=t_{0}+\left(t_{f}-t_{0}\right) \tau
$$

where $m$ is the number of grid points and $\tau_{0}=0<\tau_{1}<\cdots<$ $\tau_{m-1}<\tau_{m}=1$ is the equidistantly discretized interval with step size $h=1 / \mathrm{m}$. In the sequel $t_{0}=0$ and the time grid will be $t_{i}=i h t_{f}$ for $i=0,1, \ldots, m$.

\subsection{Direct multiple shooting}

The time-optimal control problem of the 2WD vehicle motion is solved by using the direct multiple shooting method. The purpose of this technique is to transform the DNOCP problem (9) into a finite dimensional nonlinear optimization program by discretization of the state and control functions on the time grid, first described in [20] and [21].

The origin of the strategy lies in the observation that for unstable or weakly damped systems the ODE solvers may have important errors which can further increase during the numerical optimization. Similarly, the initial and final values of the trajectory are often only partially defined, thus it is an extensive problem finding feasible initial solutions for starting the numerical optimization.

Hence, perturbations were introduced in the initial state at the boundaries of the time intervals in the grid to obtain by force, i.e. by appropriately chosen additional equality constraints, that the entire state trajectory becomes a continuous solution in the progress of the numerical optimization.

In the sequel, the control inputs are considered to be piecewise constant functions and the time grid is assumed to be the same for both multiple shooting and control. Consider the grid $G_{m}$ with subintervals $\left[t_{i}, t_{i+1}\right], i=0, \ldots, \mathrm{m}-1$. On each interval $\left[t_{i}, t_{i+1}\right]$ of the grid the solution $x_{i}(t)$ of the initial value problem (IVP) with modified initial value $s_{i}$ has to be found which satisfies

$$
\begin{aligned}
\dot{x}_{i}(t) & =f\left(t, x_{i}(t), q_{i}\right), \quad \forall t \in\left[t_{i}, t_{i+1}\right] \\
x_{i}\left(t_{i}\right) & =s_{i}
\end{aligned}
$$

where $q_{i}$ is the constant control signal in the actual time interval.

This means that an initial value is shot out and the solution is determined that belongs to it in the interval. Denote with $x_{i}\left(t, s_{i}, q_{i}\right)$ the solution of the IVP. Notice that for the integration a further subdivision of the interval $\left[t_{i}, t_{i+1}\right]$ has 
been introduced which was chosen to $n=20$ in the application independently of the number of grid points $m$.

As a consequence, separate solutions can be obtained for each interval that are not necessarily connected continuously at the boundaries of the intervals. Hence, an additional equality constraint has to be introduced for each interval guaranteeing continuity of the entire solution:

$$
s_{i+1}-x_{i}\left(t_{i+1}, s_{i}, q_{i}\right)=0, \quad i=0, \ldots, m-1
$$

Subsequently $s=\left(s_{0}, \ldots, S_{m}\right)^{T} \in R^{n_{x}(m+1)}$ further discrete variables have to be optimized.

Let us define $z=\left(t_{f}, s_{0}, \ldots, s_{m}, q_{0}, \ldots, q_{m-1}\right)$, then the time optimal control problem is of type

$$
\min _{z} F(z) \quad \text { s.t. } \quad G(z) \leq 0, \quad H(z)=0
$$

\subsection{Calculations of gradients and Jacobians}

The applicability of OPTI needs the gradient $F^{\prime}$ of the objective function and the Jacobians $G^{\prime}$ and $H^{\prime}$ of the constraints.

Let us here consider only the condition of trajectory joining as the most complex problem of computing the derivatives of the intermediate state in trajectory joining constraints. The derivative of nonlinear equality constraint (13) by $s_{i+1}$ is simple, however the derivative of $x\left(t_{i}\right)$ by $s_{i}$ is much more complicated as will be illustrated.

The question is how to determine the derivatives of $x(t, s, q)$ by the initial condition $s$, i.e. the shooting, and the parameter $q$, i.e. the actual control.

$$
\begin{aligned}
S(t) & :=\frac{d x(t)}{d s} \Rightarrow \\
\frac{d S}{d t} & =\frac{d}{d t} \frac{d x(t)}{d s}=\frac{d}{d s} \frac{d x(t)}{d t}=\frac{d}{d s} f(t, x(t), q) \\
& =f_{x}(t, x(t), q) \frac{d x(t)}{d s} \\
\dot{S}(t) & =f_{x}(t, x(t), q) S(t), \quad S(0)=I \\
Q(t) & :=\frac{d x(t)}{d q} \Rightarrow \\
\frac{d Q}{d t}= & \frac{d}{d t} \frac{d x(t)}{d q}=\frac{d}{d q} \frac{d x(t)}{d t}=\frac{d}{d q} f(t, x(t), q) \\
= & f_{x}(t, x(t), q) \frac{d x(t)}{d q}+f_{q}(t, x(t), q) \\
\dot{Q}(t) & =f_{x}(t, x(t), q) Q(t)+f_{q}(t, x(t), q), \quad Q(0)=0
\end{aligned}
$$

The results can be collected in the following matrix differential equation:

$$
\begin{gathered}
\underbrace{\left[\begin{array}{cc}
\dot{S}(t) & \dot{Q}(t) \\
0 & 0
\end{array}\right]}_{W}=\left[\begin{array}{cc}
f_{x}(t, x(t), q) & f_{q}(t, x(t), q) \\
0 & 0
\end{array}\right] \times \\
\underbrace{\left[\begin{array}{cc}
S(t) & Q(t) \\
0 & I
\end{array}\right]}_{W}, \quad W(0)=\left[\begin{array}{ll}
I & 0 \\
0 & I
\end{array}\right]
\end{gathered}
$$

Notice that $x(t)$ and $W(t)$ should be integrated numerically between $t_{i}$ and $t_{i+1}$. Because of the special structure of the matrix ODE, the Runge-Kutta method RK4 can be applied in matrix form.

\subsection{Results of DNOCP}

In this section the numerical results of the time optimal control problem are presented. The algorithm described in Section 3.2-3.3 was implemented in MATLAB with the software package OPTI. The NLP problem was solved by using IPOPT with interior point filtered line search method. The computation of the gradients and Jacobians are time consuming due to the large number of optimization variables, hence all of them were implemented in sparse forms.

The computation were performed on a Windows 7 x64 based PC equipped with Intel Core i5 3.30 GHz processor and $8 \mathrm{~GB}$ of RAM. The optimization subproblems were solved to a tolerance level of $10^{-6}$. Numerical results were computed for grid points $m=80$ and 160 . Table 2 gives an overview of the number of variables and constraints (terminal, equality, inequality, lower and upper bound).

Tab. 2. Dimensions of $2 \mathrm{WD}$ optimization problem

\begin{tabular}{ccccccc}
\hline & $\mathrm{N}_{\text {var }}$ & $\mathrm{N}_{\text {term }}$ & $\mathrm{N}_{\text {eq }}$ & $\mathrm{N}_{\text {ineq }}$ & $\mathrm{N}_{\text {bound }}$ & $\mathrm{N}_{\text {sum }}$ \\
\hline$m$ & $10 m+8$ & 8 & $7 m$ & $2 m+2$ & $20 m+16$ & $29 m+26$ \\
80 & 808 & 8 & 560 & 162 & 1616 & 2346 \\
160 & 1608 & 8 & 1120 & 322 & 3216 & 4666 \\
\hline
\end{tabular}

The optimal paths, state trajectories and control inputs of the double lane changing maneuver are shown in Fig. 2. As it can be seen, the solution of the discretized motion of the car satisfies all path, state and control input constraints.

The vehicle accelerates in the entire time interval as it was expected for time optimal solution. The resulting final time to take the maneuver are $t_{f}=5.5180 \mathrm{~s}$ and $t_{f}=5.54874 \mathrm{~s}$ for grid points $m=80$ and $m=160$ respectively.

From a good initial guess, it took approximately 10-30 iterations to find the optimal solution in case of $m=160$ grid points. The average computational time of one iteration was around $200-500$ msec.

\section{Optimal wheel force distribution}

\subsection{Four-wheel driven vehicle model}

The 4WD front-steered vehicle model used is shown in Fig. 3. It is assumed that the front $(F L$ and $F R)$ and rear $(R L$ and $R R)$ wheels are identical and the length of the half front $\left(b_{f}\right)$ and the rear $\left(b_{r}\right)$ axles are the same. The total $F_{x}$ and $F_{y}$ forces can be expressed in the global reference frame as the function of the wheels' longitudinal $(l)$ and lateral $(t)$ forces: 

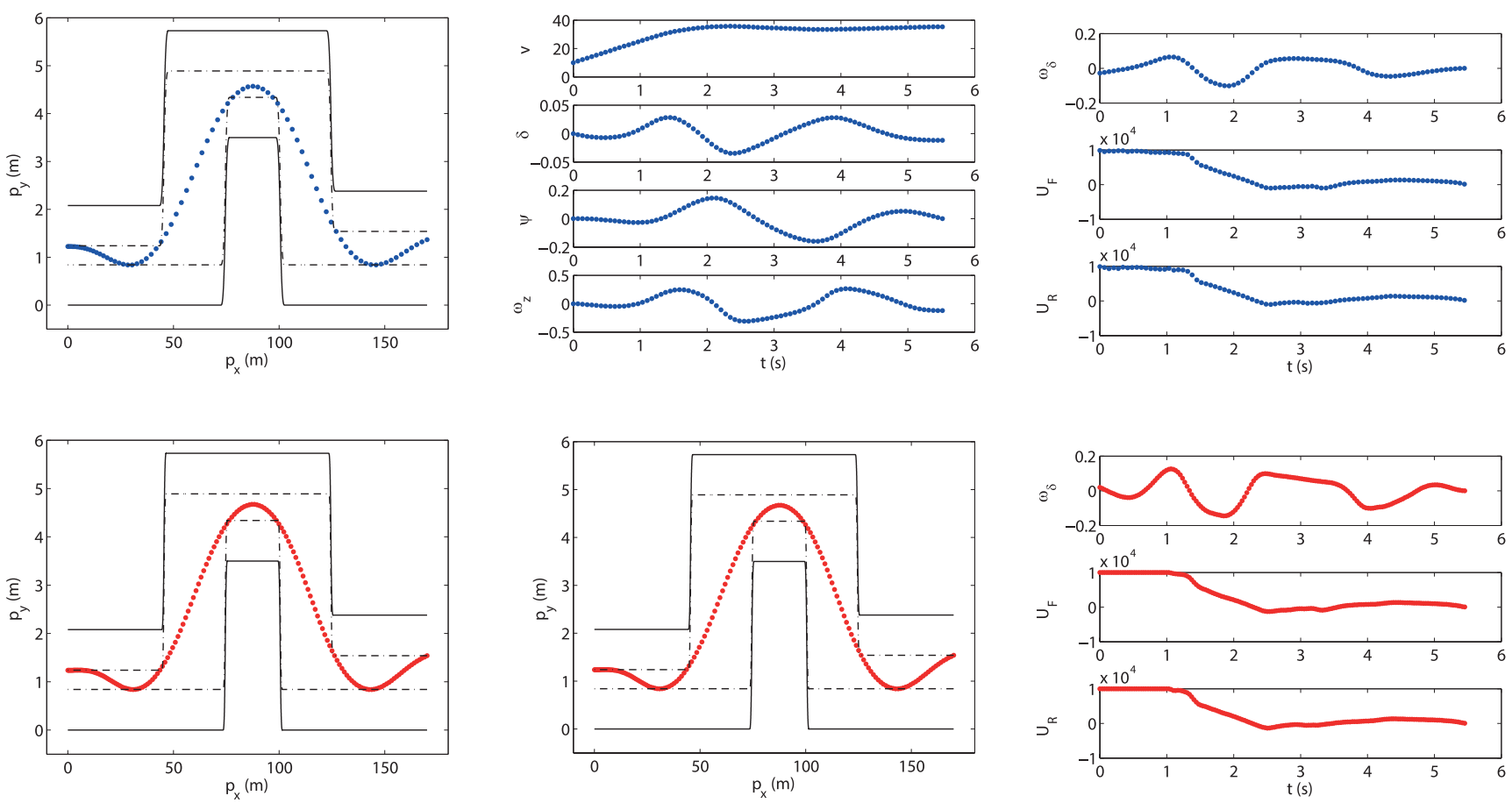

Fig. 2. Solution of $2 \mathrm{WD}$ nonlinear optimization problem for grid points $m=80$ (top row) and 160 (bottom row)

$$
\begin{aligned}
F_{x}= & \left(F_{l F L}+F_{l F R}\right) C_{\delta_{w}}-\left(F_{t F L}+F_{t F R}\right) S_{\delta_{w}} \\
& +F_{l R L}+F_{l R R}-F_{A x} \\
F_{y}= & \left(F_{l F L}+F_{l F R}\right) S_{\delta_{w}}+\left(F_{t F L}+F_{t F R}\right) C_{\delta_{w}} \\
& +F_{t R L}+F_{t R R}-F_{A y}
\end{aligned}
$$

where $C_{\delta w}=\cos \delta_{w}$ and $S_{\delta w}=\sin \delta_{w}$ are used for shorthand. Therefore, the torque about the $z$-axis can be formed

$$
\begin{aligned}
M_{z}= & b_{f}\left[S_{\delta_{w}}\left(F_{t F L}-F_{t F R}\right)-C_{\delta_{w}}\left(F_{l F L}-F_{I F R}\right)\right] \\
& +l_{f}\left[S_{\delta_{w}}\left(F_{l F L}+F_{I F R}\right)+C_{\delta_{w}}\left(F_{t F L}+F_{t F R}\right)\right] \\
& -b_{r}\left(F_{I R L}-F_{I R R}\right)-l_{r}\left(F_{t R L}+F_{t R R}\right)
\end{aligned}
$$

Since $b_{f}=b_{r}$ the rolling resistances of the front and rear wheels equal to $F_{r F} / 2$ and $F_{r R} / 2$ respectively. Thus, the longitudinal wheel forces can be determined similarly to (4) for each wheel

$$
F_{l j}=U_{j}-F_{r j}, \quad j=\{F L, F R, R L, R R\}
$$

where $U_{j}$ is the longitudinal control force as input of the vehicle.

The slip angles are computed from the velocities of the wheels as follows

$$
\begin{gathered}
v_{F L, F R}=\left(v C_{\beta} \mp b_{f} \omega_{z}, v_{S} \beta+l_{f} \omega_{z}\right)^{T} \\
v_{R L, R R}=\left(v C_{\beta} \mp b_{r} \omega_{z}, v_{S} \beta-l_{r} \omega_{z}\right)^{T} \\
\alpha_{F L}=\delta_{w}-\arctan \left(v_{F L_{y}} / v_{F L_{x}}\right) \\
\alpha_{F R}=\delta_{w}-\arctan \left(v_{F R_{y}} / v_{F R_{x}}\right) \\
\alpha_{R L}=-\arctan \left(v_{R L_{y}} / v_{R L_{x}}\right)
\end{gathered}
$$

$$
\alpha_{R R}=-\arctan \left(v_{R R_{y}} / v_{R R_{x}}\right)
$$

The front and rear transversal wheel forces can be determined by equations (21) and the magic formula (7) with peak parameter $D_{f} / 2$ and $D_{r} / 2$ respectively.

The dynamic model of the 4WD car can be given in the following form

$$
\begin{gathered}
\dot{p}_{x}=v C_{\psi+\beta} \\
\dot{p}_{y}=v S_{\psi+\beta} \\
\dot{v}=\frac{1}{m}\left(C_{\beta} F_{x}+S_{\beta} F_{y}\right) \\
\dot{\delta}_{w}=\omega_{\delta} \\
\dot{\beta}=\frac{1}{m v}\left(C_{\beta} F_{y}-S_{\beta} F_{x}\right)-\omega_{z} \\
\dot{\psi}=\omega_{z} \\
\dot{\omega}_{z}=\frac{M_{z}}{I_{z z}}
\end{gathered}
$$

with state and control variable vectors of

$$
\begin{aligned}
& x=\left(p_{x}, p_{y}, v, \delta_{w}, \beta, \psi, \omega_{z}\right)^{T} \\
& u=\left(\omega_{\delta}, U_{F L}, U_{F R}, U_{R L}, U_{R R}\right)^{T}
\end{aligned}
$$

\subsection{Problem formulation}

The aim is to find the 4WD control forces such that the resulting motion of the center of gravity is similar to the one of the 2 WD's. This is achieved by distributing the 2WD control forces on each wheel individually such that the errors of the global 
forces and torques (vector sum of all tire forces and torques) acting on the different $\mathrm{CoG}$ points are minimized. The main reason for this is that the distributed longitudinal forces can be used as nominal controls in the moving horizon algorithm.

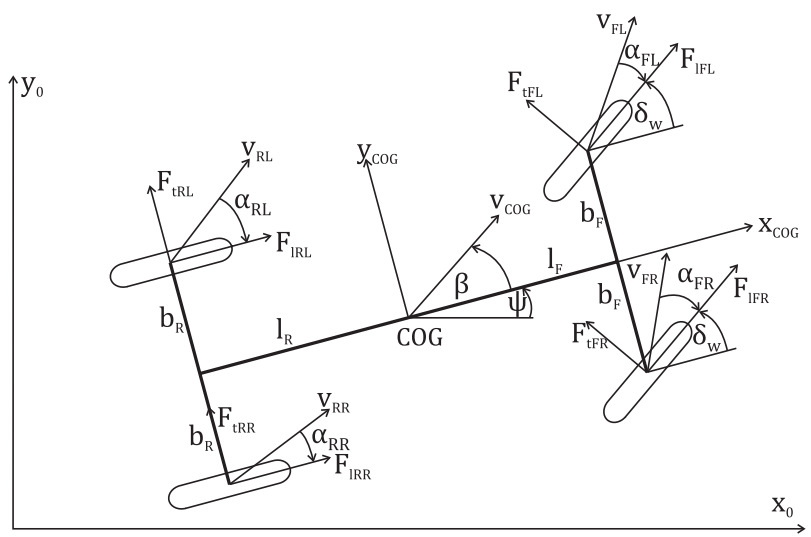

Fig. 3. Four-wheel driven model of the vehicle

Define the control vector $z_{w}=\left(U_{F L}, U_{F R}, U_{R L}, U_{R R}\right)^{T}$ and the global forces and torque of the 2WD time optimal solution $F_{x}^{*}, F_{y}^{*}, M_{z}^{*}$. The optimal wheel force distribution can be formulated as a static, linearly constrained quadratic programming (LCQP) problem

$$
\begin{gathered}
\min _{z_{w}} c_{1}\left(F_{x}-F_{x}^{*}\right)^{2}+c_{2}\left(F_{y}-F_{y}^{*}\right)^{2}+c_{3}\left(M_{z}-M_{z}^{*}\right)^{2} \\
\text { s.t. } \quad z_{w 3}=\eta z_{w 1} \cos \delta_{w} \\
z_{w 4}=\eta z_{w 2} \cos \delta_{w} \\
z_{w} \in[-5000,5000]^{4}
\end{gathered}
$$

where $c_{1}, c_{2}$ and $c_{3}$ are positive weighting constants. The objective function (23a) minimizes the errors in a least-squares sense. The constraints (23b)-(23c) defines the longitudinal force ratio of the $F L, R L$ and $F R, R R$ wheels by the constant parameter $\eta$. Here, the states such as $\delta_{w}$, are from the actual 4 WD states.

\section{Optimal Force Distribution Algorithm}

For every $i=0, \ldots, m-1$, grid points repeat the following steps:

1. Calculate the constant forces and torque $F_{x, i}^{*}, F_{y, i}^{*}, M_{z, i}^{*}$ for the actual grid point using the time optimal solution $x_{2 W, i}$. If $i=0$ set the initial state $x_{4 W, 0}:=x_{2 W, 0}$.

2. Determine the optimal solution $z_{w}$ by solving the quadratic programming defined in Eq.(23).

3. Use RK4 scheme to integrate $\dot{x}_{4 W}=f_{4 W}\left(x_{4 W}, z_{w}\right)$ to obtain $x_{4 W, i+1}$. Set $x_{4 W, i}:=x_{4 W, i+1}$.

\subsection{Results of the LCQP problem}

The resulting motion with the original $2 \mathrm{WD}$ time optimal and the LCQP optimally distributed control for $\eta=1$ is shown in Fig. 4. The weights were chosen $c_{1}=4 \mathrm{e}-5, c_{2}=4 \mathrm{e}-5$ and $c_{3}=$ 6e-4. The comparison of the 2WD optimal forces $F_{x}^{*}, F_{y}^{*}$ and moment $M_{z}^{*}$ with the LCQP optimally distributed 4WD forces $F_{x}, F_{y}$, and moment $M_{z}$ together with the reached RMSE error measures are shown in Fig. 5. The original and optimally distributed longitudinal control forces are given in Fig. 6 being in accord with the chosen value of $\eta=1$. It can be seen that the LCQP distribution is efficient. Notice that the tire slip angles and the transversal forces are newly computed for the 4WD case because the velocities are changed.

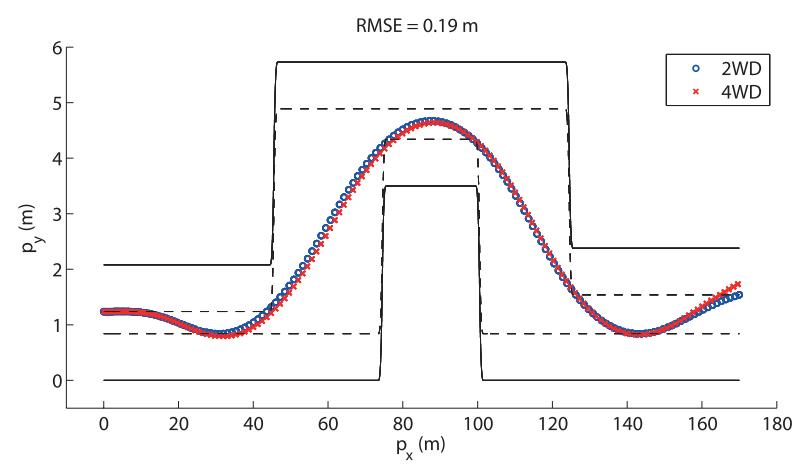

Fig. 4. Resulting motion with the original and the distributed controls
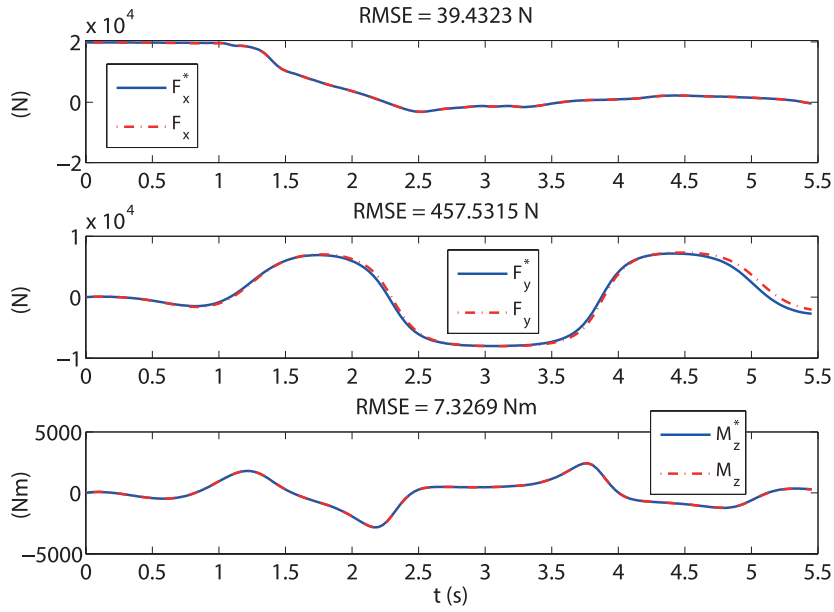

Fig. 5. Original and LCQP approximation of forces and torque with the reached RMSE errors
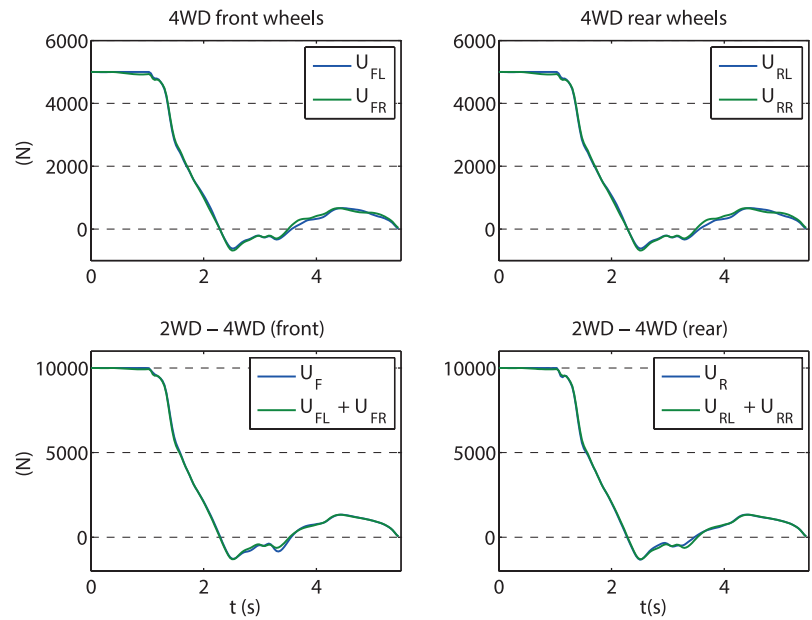

Fig. 6. Original and optimally distributed longitudinal control forces 


\section{Model predictive control of 4WD vehicle}

In this section the moving horizon model predictive control (MPC) algorithm of the four-in-wheel-motors driven vehicle is presented.

In general, model predictive control optimizes a cost function in open loop using the prediction of the system future behavior based on the dynamic model. It determines the future optimal control sequence within the horizon, applies the first element of the control sequence in closed loop to the real system, and repeats these steps for the new horizon which is the previous one shifted by the sampling time $T$. An open question is the stability of the closed loop nonlinear system, but the chance for stability is increasing with increasing the horizon length $N$, see [22].

If the nonlinear dynamic model is used for prediction, then usually a nonlinear optimization has to be solved in real-time which is time-critical for fast systems. Hence, linearization around the prescribed nominal trajectory may be suggested and optimization of the perturbations using quadratic cost and analytically manageable end-constraints can be used. If the control has been determined in the previous horizon and the errors are small relative to the prescribed path, then this control can be used as nominal control in the actual horizon, for which the optimal perturbations have to be found that decrease the path error. Clearly, a good initial nominal control is needed for the very first horizon.

\subsection{Nominal values and perturbations}

Denote with $\left\{u_{0}, u_{1}, \ldots, u_{N-1}\right\}$ the nominal control sequence within the horizon and with $\left\{x_{0}, x_{1}, \ldots, x_{N}\right\}$ the nominal state sequence belonging to it computed from the nonlinear vehicle model $\dot{x}=f_{4 W}(x, u)$, described by (22). Let the initial state $\hat{x}_{0}$ be different from $x_{0}$, i.e. an estimation of the initial state, and define the state and control perturbations be $\delta x_{0}=\hat{x}_{0}-x_{0}, \delta x_{1}, \ldots, \delta x_{N}$ and $\delta u_{0}, \delta x_{1}, \ldots, \delta x_{N}$ respectively.

The 4 WD nonlinear dynamic model can be discretized by using the RK4 scheme with sampling time $T$. This approximated system then can be linearized around the nominal sequences. The resulting linear time-varying system (LTV) for the perturbations can be given in the form

$$
\begin{gathered}
\delta x_{i+1}=A_{i} \delta x_{i}+B_{i} \delta u_{i} \\
\delta y_{i}=C \delta x_{i}
\end{gathered}
$$

where $A_{i}=\left.\frac{\partial f_{4 W}}{\partial x}\right|_{\left(x_{i}, u_{i}\right)}, \quad B_{i}=\left.\frac{\partial f_{4 W}}{\partial u}\right|_{\left(x_{i}, u_{i}\right)}$ and $C$ is a constant selector matrix. The output is assumed to be $y=\left(p_{x}, p_{y}, \delta_{w}, \psi\right)^{T}$.

Let $\left\{y_{i}=C x_{i}\right\}$ be the computed nominal output sequence for $i=0, \ldots, N$ and $\left\{y_{d 0}, y_{d 1}, \ldots, y_{d N}\right\}$ be the desired output sequence. Then, the output errors due to the perturbations are

$$
y_{d i}-C\left(x_{i}+\delta x_{i}\right)=e_{i}-\delta y_{i}, \quad i=0, \ldots, N
$$

The transients of the perturbed LTV system can be determined as a function of the perturbation of the initial state and the perturbations of the control inputs in the actual horizon by iteratively using (24) and (25):

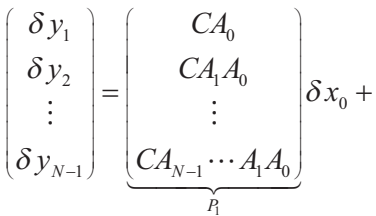

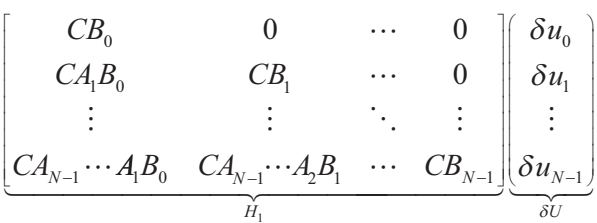$$
\delta y_{N}=P_{2} \delta x_{0}+H_{2} \delta U
$$

\subsection{MPC optimization using end constraints}

The goal is to find the control perturbation $\delta U$ such that error from the desired trajectory is minimized at the end of the horizon. This problem can be formulated as a quadratic programming structure with equality constraint. The MPC optimization problem reads as

$$
\begin{gathered}
\min _{\delta U} \quad \frac{1}{2} \sum_{i=1}^{N-1}\left\|e_{i}-\delta y_{i}\right\|_{Q}^{2}+\frac{1}{2} \sum_{i=0}^{N-1}\left\|\delta u_{i}\right\|_{\Lambda}^{2} \\
\text { s.t. } \quad e_{N}-\delta y_{N}=0
\end{gathered}
$$

where (28) is the objective function that penalizes both output errors and large deviations from the nominal control and the constraint (29) assures zero output error at the end of the horizon. The order of the input perturbations and output errors can be influenced by the weighting matrices $A$ and $Q$ respectively.

\subsection{Integral control}

It is possible to incorporate integrator in the controller using augmented state $\delta x_{i}=\left(\delta x_{i}^{T}, \delta u_{i-1}^{T}\right)$ and $\delta u_{\mathrm{i}}=\delta u_{i-1}+\delta r_{i}$ where the change of the control $\delta r_{i}$ has to be optimized. Substituting

$$
A_{i}:=\left[\begin{array}{cc}
A_{i} & B_{i} \\
0 & I
\end{array}\right] \quad B_{i}:=\left[\begin{array}{c}
B_{i} \\
I
\end{array}\right]
$$

the earlier results remain valid in the new variables. However, in this case, $\delta R$ is the optimal change of the control differences and the optimal $\delta U$ is the cumulative sum of $\delta R$.

\subsection{MPC algorithm}

The solution of the MPC optimization problem is derived by the Lagrange multiplier method. Because $\lambda$ is reserved for weighting factor, the Lagrange multiplier will be denoted by $\mu$.

First, the objective function is augmented with the constraint multiplied by the vector Lagrange multiplier, then it is differentiated and the derivative is set to zero. The solution $\delta U(\mu)$ is substituted into the constraints equation in a linear way. From the resulting linear equation, $\mu$ can be expressed and 
plugged into $\delta U(\mu)$ that results the optimal control perturbation sequence. Details of the calculations can be found in [22].

The final result can be summarized in the following form for non-integral control:

$$
\begin{gathered}
L_{1}=H_{1}^{T} Q_{e} H_{1}+\Lambda_{e} \\
L_{\mu}=Q_{N} H_{2} L_{1}^{-1} H_{2}^{T} Q_{N} \\
R=H_{2}^{T} Q_{N} L_{\mu}^{-1} Q_{N} \\
S=H_{1}^{T} Q_{e} P_{1} \\
\delta U=L_{1}^{-1}\left\{R e_{N}+\left(I-R H_{2} L_{1}^{-1}\right) H_{1}^{T} Q_{e} E\right. \\
\left.-\left[S+R\left(P_{2}-H_{2} L_{1}^{-1} S\right)\right] \delta x_{0}\right\}
\end{gathered}
$$

where $Q_{e}$ and $\Lambda_{e}$ are the block diagonal matrices by $N-1$ times of $Q$ and $\Lambda$ respectively and $E=\left(e_{1}^{T}, e_{2}^{T}, \ldots, e_{N-1}^{T}\right)$ is the vector of the nominal output errors. The closed loop control is $u_{0}+\delta u_{0}$ where $u_{0}$ is the nominal control and $\delta u_{0}$ is the first element of the open loop optimal control sequence $\delta U$.

In case of integral control (3.5) is used to obtain $\delta R$ and the optimal $\delta U$ is the cumulative sum of $\delta R$.

\section{MPC Integral Control Algorithm}

In every horizon, the following steps are repeated:

1. From the initial state $x_{0}$ and the nominal control sequence $\left\{\mathrm{u}_{i}\right\}$, calculate the nominal state sequence $\left\{x_{i}\right\}$ using the discrete nonlinear dynamics of the system. Here, $x_{0}$ is coming from the shifted previous horizon and can differ from the estimated state $\hat{x}_{0}$. In case of the very first horizon, use the first $N$ elements of the time optimal solution $\omega_{\delta}$ and the LCQP optimally distributed forces as nominal controls.

2. Determine the matrices $A_{i}$ and $B_{i}$ for $i=0, \ldots, N-1$ using the nominal states, controls and Eq. (30).

3. Set up $P_{1}, P_{2}$ and $H_{1}, H_{2}$ matrices and compute the state $\left\{\delta x_{i}\right\}$ and output perturbations $\left\{\delta y_{i}\right\}$ by Eq. (27).

4. From the 2WD time optimal solution, choose the desired output sequence $\left\{y_{d i}=\left(p_{x i}, p_{y i}, \delta_{w i}, \psi_{i}\right)^{T}\right\}$ and use (26) to determine the output error vector $E$.

5. Compute the optimal change of the control differences $\delta R$ by Eqs. (31)-(35) using $\delta x_{0}=\hat{x}_{0}-x_{0}$ where $\hat{x}_{0}$ is the estimated state. Calculate $\delta U$ as the cumulative sum of $\delta R$. Update the optimal control sequence $U:=U+\delta U$ and apply its first element $u_{0}$ in closed loop.

6. Set the new initial state $x_{0}$ for the next horizon as the result of the applied control $u_{0}$. Initialize the nominal control sequence for the next horizon using the optimal control $U$ as $\left\{u_{1}, u_{2}, \ldots, u_{N-1}, u_{N-1}\right\}$, i.e. shift it by one and repeat the last element.

More clever methods exist for the choice of $u_{N-1}$ instead of the repetition [22].

\subsection{Results of the MPC integral control}

The MPC control algorithm was implemented by using the $m$ $=160$ grid point solution of the time optimal control problem. Sampling time $T=\frac{t_{f}}{m}$ and horizon $N=40$ were chosen. The weighting matrices were set as follows:

$$
\begin{aligned}
\Lambda_{i} & =\operatorname{diag}\left(2000,2 \cdot 10^{-4}, 2 \cdot 10^{-4}, 2 \cdot 10^{-4}, 2 \cdot 10^{-4}\right) \\
Q_{i} & =\operatorname{diag}(2,2,2000,2000) \\
Q_{N} & =\operatorname{diag}(1,1,1000,1000)
\end{aligned}
$$

The output trajectories with time optimal 2WD and the moving horizon MPC control are shown in Fig. 7. Desired output signals $y_{d}=\left(p_{x}, p_{y}, \delta_{w}, \psi\right)_{d}^{T}$ for the MPC control were considered as the result of the subset of the state variables belonging to LCQP optimal wheel force distribution. Notice the difference in the initial state for MPC. The optimally distributed nominal and the MPC control signals are compared in Fig. 8 and Fig. 9. Notice that the nominal control is used only in the very first horizon.

The results demonstrate the efficiency of the moving horizon MPC, especially the errors between the paths decay quickly and vanishes after $40 \mathrm{~m}$ in $x$-direction. The $4 \mathrm{WD}$ MPC control forces are well in their prescribed domain and the braking forces, i.e. where the longitudinal forces are negative, are also small in accord with the form and dimensions of the path. The difference between nominal and MPC steering angle derivatives decays after $0.7 \mathrm{~s}$. The derivatives of the steering angles are smooth.

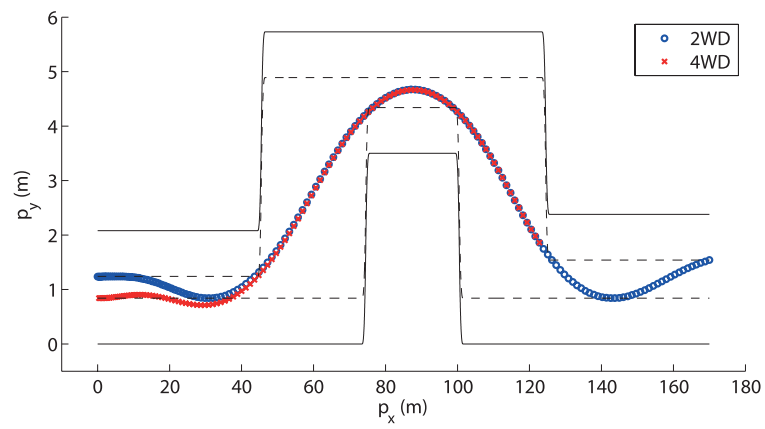

Fig. 7. Output trajectory of the MPC algorithm if the real initial state differs from the optimal one
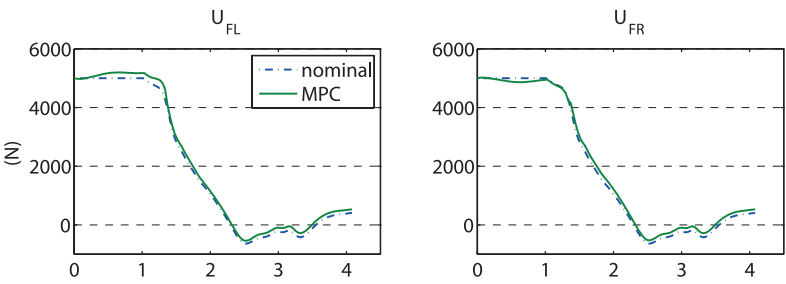

$U_{\text {RL }}$
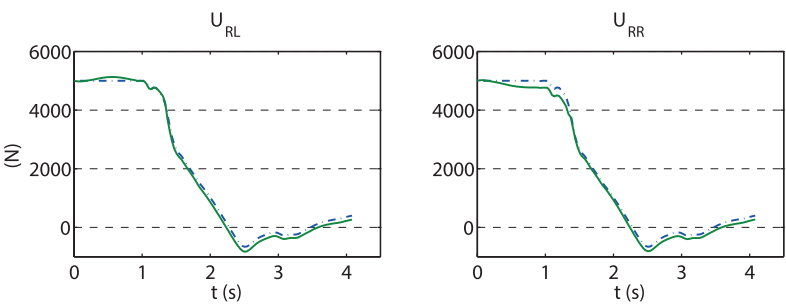

Fig. 8. Nominal and MPC control signals if the real initial state differs from the optimal one 


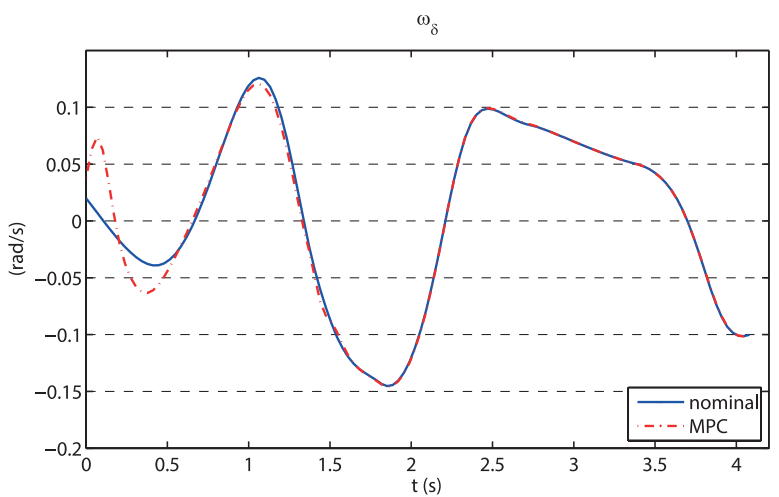

Fig. 9. MPC controlled angular velocity of the steering angle

\subsection{Collision Avoidance System (CAS)}

The number of test path parameters is $5+3=8$, hence a grid of parameters can be chosen and for all grid points (i.e. path) the time optimal control trajectory can be determined offline and stored in a database. For a given situation of lane change or collision avoidance in the presence of static obstacle the nearest solution in the database can be selected, perhaps interpolation can be involved and the control trajectory can be performed in real-time.

If the state trajectory is also stored in the database then it can be applied in the case of additional moving obstacle to check in real-time whether the obstacle avoidance can be performed or emergency braking is necessary.

\section{Conclusion}

In this paper, we investigated the extensively researched time optimal control problem for 4WD electric cars. The problem was solved by using time discretization and multiple shooting method. The approach was implemented with the aid of non-commercial software packages that substantially use the gradients of the objective function and the Jacobians of the constraints. For the latter, we presented a novel algorithm for computing the derivatives of the complex trajectory joining equations. This algorithm was given in the form of matrix differential equations whose structure allowed to compute their solution using RK4 in matrix form. Because of the large number of variables the gradients and Jacobians were performed in sparse form.

A novel method was presented for the optimal distribution of the 4WD longitudinal forces. For tolerating the differences between optimal and real initial states a moving horizon MPC method was elaborated which does not need numerical optimization.

The methods for optimal force distribution and MPC control are usable in real time. The 2WD time optimization is an offline method. However a database of optimal control trajectories over a grid of path parameters can be prepared for a general CAS system. For a given situation of lane change or collision avoidance in the presence of static obstacle the nearest solution in the database can be selected and the control trajectory can be performed in real-time using the developed optimal force distribution and MPC method.

Our solution showed competitive results to the ones obtained by closed source and state of the art commercial solvers.

Future research will concentrate on the development of a quasi time-optimal MPC control method for general paths that does not need any offline optimization and obtain the solution nearly real time.

\section{Acknowledgement}

The work was supported by the European Union and Hungary in the project TÁMOP-4.2.2.A-11/1/KONV-2012-0012: „Basic research for the development of hybrid and electric vehicles".

\section{References}

1 Max G., Lantos B. Time-optimal control of ground vehicles. in 'Proceedings of IEEE 12th International Conference on Intelligent Systems and Informatics SYSI 2014. Subotica, Serbia' (2014). DOI: $10.1109 /$ sisy.2014.6923594

2 Sager S., Kirches C., Bock H. G. Fast solution of periodic optimal control problems in automobile test-driving with gear shifts. in 'Conference on Decision and Control' (2008). DOI: $10.1109 /$ cdc.2008.4739014

3 Gerdts M. Solving mixed-integer optimal control problems by branch and bound: a case study from automobile test-driving with gear shift. Optimal Control Applications and Methods. 26 (1), pp. 1-18 (2005). DOI: $10.1002 /$ oca.751

4 Kirches C., Sager S., Bock H. G., Schlöder J. P. Time-optimal control of automobile test drives with gear shifts. in 'Optimal Control Applications and Methods' Wiley Inter Science (2009).
5 Gerdts M., Karrenberg S., Müller-Bessler B., Stock, G. Generating locally optimal trajectories for an automatically driven car. Optimization and Engineering. 10 (4), pp. 439-463 (2009). DOI: $10.1007 / \mathrm{s} 11081-008-9047-1$

6 Kirches C. Fast numerical methods for mixed-integer nonlinear-predictive control. Ph.D. dissertation, Ruprecht-Karls-University Heidelberg, Heidelberg, (2010).

7 Diehl M., Leineweber D., Schaefer A. MUSCOD-II User's Manual. Heidelberg: IWR Preprint 2001-25. (2001).

8 Currie J., Wilson D. I. OPTI: Lowering the Barrier Between Open Source Optimizers and the Industrial MATLAB User. in 'Foundations of Computer-Aided Process Operations' (eds.: Sahinidis, N. and Pinto, J.) Savannah, Georgia, (2012). 
9 Sawase K., Ushiroda Y. Improvement of vehicle dynamics by rightand left torque vectoring system in various drive trains. Mitsubishi Motors Technical Review, no. 20, pp. 14-20, 2008

10 Ono E., Hattori Y., Muragashi Y., Koibuchi K. Vehicle dynamics integrated control for fourwheel-distributed steering and four-wheeldistributed traction/braking systems. Vehicle System Dynamics, 44 (2), 139-151 (2006).

DOI: $10.1080 / 00423110500385790$

11 Klomp M. Longitudinal force distribution using quadratically constrained linear programming. Vehicle System Dynamics, 49 (12), pp. 1823-1836 (2011).

DOI: $10.1080 / 00423114.2010 .545131$

12 Rajamani R. Vehicle Dynamics and Control. Springer, (2006).

13 Pacejka H. B. Tyre and Vehicle Dynamics. ser. Automotive engineering. Butterworth-Heinemann, (2006).

14 Pacejka H. B., Bakker, E. The magic formula tyremodel in '1st Tyre Colloquium, SupplementtoVehicle Systems Dynamics' Delft, The Netherlands, Vol, 21, (1993).

15 Manfred M., Wallentowitz H. Dynamik der Kraftahrzeuge. Springer, (2004).

16 Sager S. A benchmark library of mixed-integer optimal control problems. (2012)
17 Wächter A., Biegler L. T. On the implementation of an interior-point filter line-search algorithm for large-scale nonlinear programming. Mathematical Programming, 106 (1), pp. 25-57 (2006).

DOI: $\underline{10.1007 / \mathrm{s} 10107-004-0559-\mathrm{y}}$

18 Currie J., Wilson D. I. Industrial Informationand Control CentreOPTI Toolbox. http://www.i2c2.aut.ac.nz/Wiki/OPTI/, 2012.

19 Wächter A., Laird C. COIN-OR Project. https://projects.coin-or.org/ Ipopt, 2005.

20 Bock H. G. Numerical treatment of inverse problem sin chemical reaction kinetics. in 'Modelling of Chemical Reaction Systems, ser. Springer Series in Chemical Physics'. Springer Berlin Heidelberg, vol. 18, (1981). DOI: $10.1007 / 978-3-642-68220-9 \_8$

21 Bock H. G., Plitt K. J. A Multiple Shooting algorithm for direct solution of optimal control problems. in 'Proceedings of the 9th IFAC World Congress' Pergamon Press, Budapest (1984).

22 Lantos B., Márton L. Nonlinear Control of Vehicles and Robots. Springer, (2011).

DOI: $\underline{10.1007 / 978-1-84996-122-6}$ 